\title{
Hypothesis-driven compartment model for stirred bioreactors utilizing computational fluid dynamics and multiple $\mathrm{pH}$ sensors
}

Nørregaard, Anders; Bach, Christian; Krühne, Ulrich; Borbjerg, Ulrik; Gernaey, Krist V.

Published in:

Chemical Engineering Journal

Link to article, DOI:

10.1016/j.cej.2018.08.191

Publication date:

2018

Document Version

Peer reviewed version

Link back to DTU Orbit

Citation (APA):

Nørregaard, A., Bach, C., Krühne, U., Borbjerg, U., \& Gernaey, K. V. (2018). Hypothesis-driven compartment model for stirred bioreactors utilizing computational fluid dynamics and multiple pH sensors. Chemical Engineering Journal, 356, 161-169. https://doi.org/10.1016/j.cej.2018.08.191

\section{General rights}

Copyright and moral rights for the publications made accessible in the public portal are retained by the authors and/or other copyright owners and it is a condition of accessing publications that users recognise and abide by the legal requirements associated with these rights.

- Users may download and print one copy of any publication from the public portal for the purpose of private study or research.

- You may not further distribute the material or use it for any profit-making activity or commercial gain

- You may freely distribute the URL identifying the publication in the public portal 


\section{Accepted Manuscript}

Hypothesis-driven compartment model for stirred bioreactors utilizing computational fluid dynamics and multiple $\mathrm{pH}$ sensors

Anders Nørregaard, Christian Bach, Ulrich Krühne, Ulrik Borbjerg, Krist V. Gernaey

PII: S1385-8947(18)31671-1

DOI: https://doi.org/10.1016/j.cej.2018.08.191

Reference:

CEJ 19805

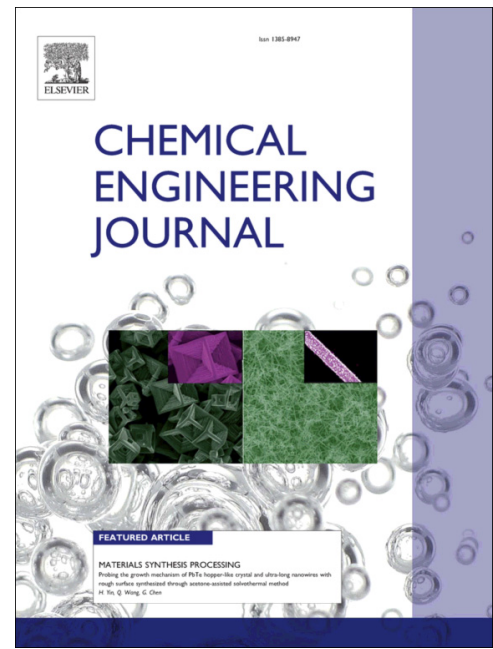

To appear in:

Chemical Engineering Journal

Received Date:

25 March 2018

Revised Date:

1 July 2018

Accepted Date:

27 August 2018

Please cite this article as: A. Nørregaard, C. Bach, U. Krühne, U. Borbjerg, K.V. Gernaey, Hypothesis-driven compartment model for stirred bioreactors utilizing computational fluid dynamics and multiple $\mathrm{pH}$ sensors, Chemical Engineering Journal (2018), doi: https://doi.org/10.1016/j.cej.2018.08.191

This is a PDF file of an unedited manuscript that has been accepted for publication. As a service to our customers we are providing this early version of the manuscript. The manuscript will undergo copyediting, typesetting, and review of the resulting proof before it is published in its final form. Please note that during the production process errors may be discovered which could affect the content, and all legal disclaimers that apply to the journal pertain. 


\section{Hypothesis-driven compartment model}

\section{for stirred bioreactors utilizing}

computational fluid dynamics and

\section{multiple pH sensors}

Authors:

Anders Nørregaard ${ }^{1 *}$, Christian Bach ${ }^{2 *}$, Ulrich Krühne ${ }^{2}$, Ulrik Borbjerg ${ }^{1}$ \& Krist V. Gernaey ${ }^{2}$

1: Novo Nordisk A/S, Bagsværd, Denmark

2: Process and Systems Engineering Center (PROSYS), Department of Chemical and Biochemical Engineering, Technical University of Denmark (DTU), Lyngby, Denmark

Keywords: Stirred bioreactor, CFD, Compartments models, Hydrodynamics, Mixing 


\section{Abstract}

The ability to understand and predict mixing behaviour in stirred bioreactors through the use of mathematical models is an effective strategy to improve or ensure the performance of a process. Historically, compartment models have been developed to facilitate the prediction of hydrodynamics and in the last decade, computational fluid dynamics (CFD) has become the standard tool to assess this in detail. Despite the continuous increase in computational capacity, performing CFD simulations of bioreactors remains a challenge in industry, where the need for simplified models is apparent due to the complex phenomena occurring at the industrial scale. The combination of CFD and compartment models has been investigated in this work using a pilot scale stirred bioreactor equipped with three Rushton disc turbines and multiple $\mathrm{pH}$ sensors as a case study. A hypothesisdriven compartmentalization strategy, comprised of five steps, has been developed and applied to the pilot scale stirred bioreactor. The compartmentalization resulted in 56 compartments with unidirectional flow between adjacent compartments. The performance of the methodology was evaluated against a data-driven compartment approach and the full CFD simulation, in terms of its ability to recreate transient tracer profiles following top and bottom feeding of the tracer. The datadriven compartment model proved to be the most accurate of the three investigated methods, whereas the hypothesis-driven compartment method had a 10-12 \% higher error on prediction. Nevertheless, the developed methodology should be considered a viable alternative to conventional CFD methods, especially when models of complex phenomena - for example multi-dimensional population balance models - are to be incorporated in the final model as well. 


\section{Nomenclature}

\begin{tabular}{|c|c|c|}
\hline Variables & Description & Unit \\
\hline$C$ & Scaled tracer concentration & {$[-]$} \\
\hline$E F$ & Exchange flow & {$[\mathrm{kg} / \mathrm{s}]$} \\
\hline$F$ & Mass flow rate & {$[\mathrm{kg} / \mathrm{s}]$} \\
\hline$F C$ & Circumferential flow rate & {$[\mathrm{kg} / \mathrm{s}]$} \\
\hline$F H$ & Axial flow rate & {$[\mathrm{kg} / \mathrm{s}]$} \\
\hline$F V$ & Radial mass flow rate & {$[\mathrm{kg} / \mathrm{s}]$} \\
\hline$H$ & Axial division & {$[\mathrm{m}]$} \\
\hline$R$ & Radial division & {$[\mathrm{m}]$} \\
\hline$t$ & Time & {$[\mathrm{s}]$} \\
\hline$t_{\text {mix }, 95 \%}$ & 95\% mixing time & {$[\mathrm{s}]$} \\
\hline$v_{p}$ & Liquid velocity & {$[\mathrm{m} / \mathrm{s}]$} \\
\hline V & Compartment volume & {$\left[\mathrm{m}^{3}\right]$} \\
\hline$W$ & Circumferential division & {$\left[{ }^{\circ}\right]$} \\
\hline$x_{1}$ & Dimension & {$[\mathrm{m}]$} \\
\hline$x_{2}$ & Dimension & {$[\mathrm{m}]$} \\
\hline$\rho$ & Liquid density & {$\left[\mathrm{kg} / \mathrm{m}^{3}\right]$} \\
\hline $\mathrm{pH}$ & Measured $\mathrm{pH}$ & {$[-]$} \\
\hline $\mathrm{pH}_{0}$ & Initial pH before pulse & {$[-]$} \\
\hline $\mathrm{pH}_{\infty}$ & Homogenous $\mathrm{pH}$ after pulse & {$[-]$} \\
\hline \multicolumn{3}{|l|}{ Indices } \\
\hline$i$ & Radial position & \\
\hline$j$ & Axial position & \\
\hline$k$ & Wedge position & \\
\hline$f$ & Feed compartment & \\
\hline
\end{tabular}

\begin{tabular}{|l|l|}
\hline Abbreviations & \\
\hline CFD & Computational fluid dynamics \\
\hline RDT & Rushton disc turbine \\
\hline RANS & Reynolds average Navier-Stoke \\
\hline RMSE & Root mean square error \\
\hline
\end{tabular}




\section{Introduction}

Characterization of mixing in stirred bioreactors is a topic that has been studied intensively for decades [1]. Knowledge of mixing can be useful in designing, scaling and troubleshooting a biotechnological process. Mixing dynamics can also be coupled to models of biochemical reactions and gas-liquid mass transfer in the reactor vessel, to simulate the effects of substrate gradients on the process [2]. Mixing is usually quantified through tracer experiments including, but not limited to, pulses of acids/bases, salts, radioactive elements or liquids at different temperatures. The pulses are measured with one or more sensors inside the vessel to quantify the distribution of the tracer over time [3]. In particular, for investigation in smaller vessels $\left(<1 \mathrm{~m}^{3}\right)$, the response of the sensor becomes an important factor, since the measurement delay can be of the same order of magnitude as the time constant of the tracer dynamics $[4,5]$. Design and completion of these experimental investigations is time consuming and requires a meticulous choice of sensors and equipment. Mathematical models can be applied to facilitate greater understanding of the phenomena involved in mixing of stirred bioreactors, which streamlines the experimental efforts to increase their impact.

Compartment models, sometimes named Network-of-Zones models, based on well-mixed tanks in series have been used as a model framework for modelling hydrodynamics [6,7]. These methods are suitable when multiple sensors are used in the vessel to determine the tracer concentration profiles at different locations, but when the number of compartments in the models becomes greater than the number of sensors, a problem of parameter identifiability can arise. Computational fluid dynamics (CFD) forms an alternative modelling approach used to simulate hydrodynamic flows in bioreactors $[8,9]$. These models show potential, but are complicated and require considerable computational resources to work, especially when the CFD model is coupled to reaction and mass transfer kinetics. As such, in the last decade mixing studies have started integrating the knowledge acquired from CFD simulations with the limited computational effort of compartment models [1012].

The scope of this article is firstly to produce a mathematical mixing model based on a hypothesis of bulk hydrodynamic flows in the bioreactor, which are determined and quantified from a CFD simulation. The resulting hypothesis-driven compartment model is a simplified mathematical system that is able to describe mixing properties and simultaneously accommodate reaction and mass transfer kinetics. The model represents a compromise between model complexity and the amount of necessary computations, making it especially attractive for process development. The second aim of the paper is the benchmarking of the hypothesis-driven compartment model against a CFD model, a 
simple compartment model and finally, experimental data acquired in a pilot scale bioreactor at Novo Nordisk A/S.

\section{Materials and methods}

Mixing behaviour was quantified using acid and base tracer experiments in a linear $\mathrm{pH}$ buffer solution. The medium was composed of $2 \mathrm{mM}$ succinic acid and $2 \mathrm{mM}$ malonic acid as proposed by Poulsen and Iversen [13], which produces a linear response between $\mathrm{pH}$ values of 3 and 6 . All experiments were conducted within this linear $\mathrm{pH}$ range. Alternating pulses of approximately $46 \% \mathrm{wt}$ $\mathrm{H}_{2} \mathrm{SO}_{4}$ or $\mathrm{KOH}$ solution were used to produce tracer response curves, which were measured with 5 $\mathrm{pH}$ electrodes (Endress + Hauser CPS471D-7211). The $\mathrm{pH}$ sensors were installed on a steel beam attached to a cooling coil in the bioreactor, and were positioned directly between two baffles. Acid and base pulses were introduced at three different heights using a system of silicone tubes fastened to the baffle and entering the reactor through the top of the vessel. The dosing and sensor positions are shown in Figure 1 b). A peristaltic pump (Watson Marlow 323S) was used to pump pH tracer solution into the vessel over a period of approximately 3 seconds. $\mathrm{pH}$ was logged with a central data logger (Yokogawa MW100) for all pH electrodes. The buffer temperature was kept constant at $25^{\circ} \mathrm{C}$ in the bioreactor throughout the experiments. Additionally, the experiments were carried out at a working volume that ensured all three Rushton Disc Turbines (RDTs) in the bioreactor remained submerged. A total of 36 tracer additions were conducted, testing combinations of three agitation speeds and three dosing points, with each combination being performed in quadruplicate. Although the mixing models investigated in this work are aimed towards aerobic fermentation processes, this article only addresses non-aerated mixing, to limit the complexity of the data set and the subsequent simulations.

Individual mixing profiles were normalized from 0 to 1 , representing the initial $\mathrm{pH}$ in the vessel before the pulse is added and the final $\mathrm{pH}$ after homogenization, respectively, as described in Equation (1.

$$
C(t)=\frac{p H(t)-p H_{0}}{p H_{\infty}-p H_{0}}
$$

where $\mathrm{C}(\mathrm{t})$ is the normalized $\mathrm{pH}$ at time $\mathrm{t}, \mathrm{pH}_{0}$ is the $\mathrm{pH}$ before the pulse is added and $\mathrm{pH}_{\infty}$ is the steady state $\mathrm{pH}$ after pulse addition. The measured $\mathrm{pH}$ was not corrected for the time delay of the $\mathrm{pH}$ sensor, making it a potential source of error in the experimental data. The $95 \%$ response time of 
the CPS471D-7211 pH sensors were determined experimentally with a step change of approximately 5 seconds.

Figure 1 a) displays an example of 4 repeated mixing tracer profiles with top dosing. The data is plotted against dimensionless time $(\tau)$, normalized towards the $95 \%$ mixing time using Equation $(2$, to maintain confidentiality.

$$
\tau=\frac{t}{t_{95 \% \operatorname{mix}}}
$$

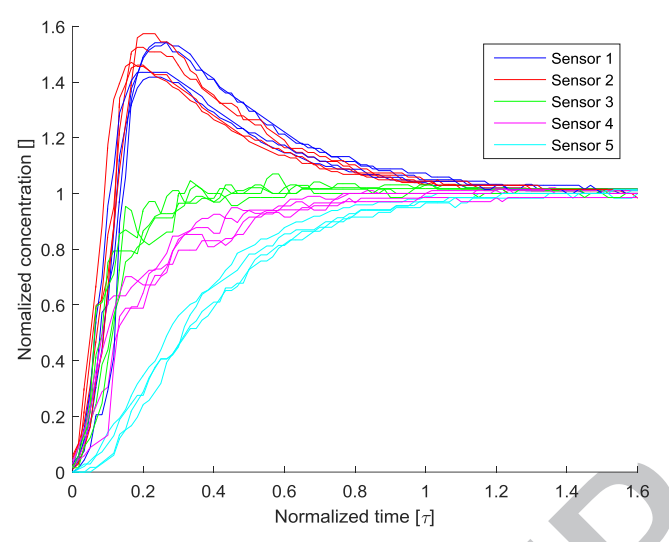

a)

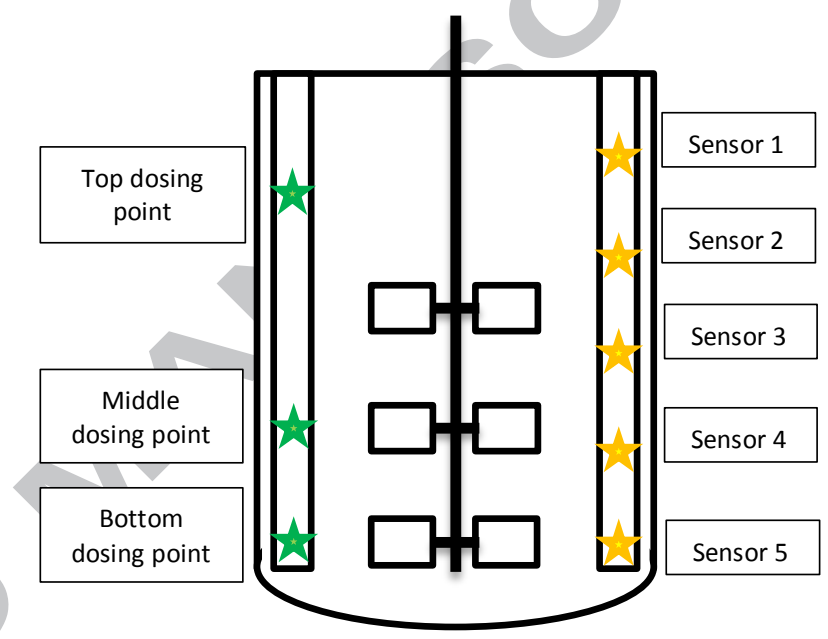

b)

Figure 1: a) Example of a normalized mixing profile measured with $5 \mathrm{pH}$ electrodes. The time axis is normalized to maintain confidentiality. b) Diagram of the bioreactor used as experimental basis for the work. The vessel is equipped with three Rushton Disc Turbines (RDT), four baffles and a cooling coil (not shown). Green stars indicate dosing positions and yellow stars indicate the $\mathrm{pH}$ sensors.

\section{4+1 compartment model}

It has long been established that RDT impellers have a tendency of producing axial separation of the liquid flow in a bioreactor. Thus, a $3 \times$ RDT configuration should divide the vessel into four well-mixed zones [14]. With this in mind, a four-compartment model was used as the simplest reference model for this study. The model is highly data-driven and thus requires large amounts of experimental data, however, it also requires the least amount of calculations and iteration to work properly, i.e. solution of this model is relatively straightforward. The model geometry was taken directly from the vessel dimensions and impeller positions, while four exchange flows between compartments were estimated with an optimization algorithm (fmincon, matlab R2015b) to fit the experimental data. A fifth pseudo compartment was added to the model to help simulate the addition of the tracer by improving the ability of the model to mimic the process of the $\mathrm{pH}$ tracer being mixed into the bulk 
flow in the vessel. The feeding compartment is assumed to be small $(0.1 \mathrm{~L})$ and coupled directly to the compartment closest to the dosing point with an exchange flow. The $4+1$ compartment model is described in detail in the Supplementary Information. The parameter estimation was carried out with a single set of exchange flows to fit experimental data from both bottom and top dosing. This was done to improve the robustness of the model.

\section{CFD flow characterization}

A CFD simulation was performed for each of the different agitation speeds using the commercial CFD software ANSYS CFX, version 16.1. Each simulation was carried out as a single phase RANS simulation, where turbulence was accounted for using the standard $\kappa-\epsilon$ model. The impeller motion was captured using a transient frozen rotor, otherwise known as a sliding mesh boundary condition, which has proven applicable for mixing time determination in previous studies $[11,15]$. Discretization of the domain was done using a hexahedral mesh configured in ICEM CFD 16, and consisted of 734,000 elements in total. The full geometry was simulated in order to capture the spatial effects of measuring and adding tracer in different locations in the vessel. A scalar was used to determine tracer dissipation and simulate the addition of acid or base in the solution. The tracer addition was based on an assumption of an ideal pulse over three seconds.

\section{Hypothesis driven $56+1$ model}

The novel hypothesis-driven compartment model developed in this work relies on evaluating circumferential, axial and radial bulk flow at different locations in the vessel. The compartments are defined as volumes with unidirectional flow at each surface in either the radial, axial or circumferential direction. This definition limits the degrees of freedom of the generated compartments because each compartment must have two surfaces in the axial, radial and circumferential direction. Expanding this definition to other coordinate systems is outside the scope of this work, and is subject to further research. The methodology, illustrated in Figure 2, is described in detail in the following sections using the pilot scale bioreactor from Novo Nordisk $A / S$ as a case study. 
Step 1: Axial division

Place multiple vertical lines

in geometry

Evaluate $v_{R a d}$ along lines

Step 2: Axial division

Identify axial division at $U_{\text {Rad }}=0$.

Step 3: Radial division

Place horizontal lines at axial

divisions

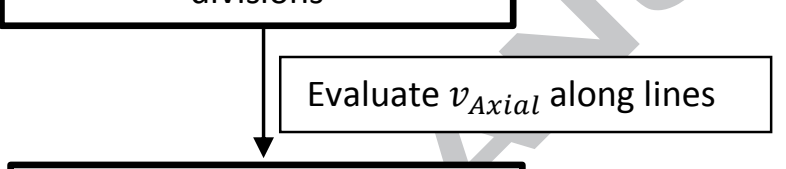

Step 4: Radial division

Determine radial division at

$\mathrm{U}_{\text {Axial }}=0$.

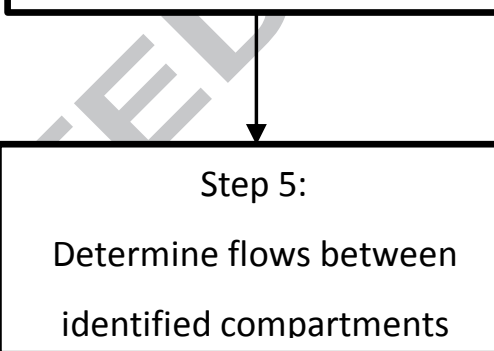

Figure 2: Schematic workflow of the compartmentalization methodology, which enables identification of individual compartments in a bioreactor

\section{Step 1 - Axial division: Place multiple vertical lines in geometry}

The first step of the methodology entails the placement of a series of vertical lines in the geometry of the CFD model. The vertical lines are placed at multiple locations, as shown in Figure 3 a), to ensure the axial division is as global as possible. Furthermore, having multiple sample points helps to determine the spatial uncertainty of the compartments. 


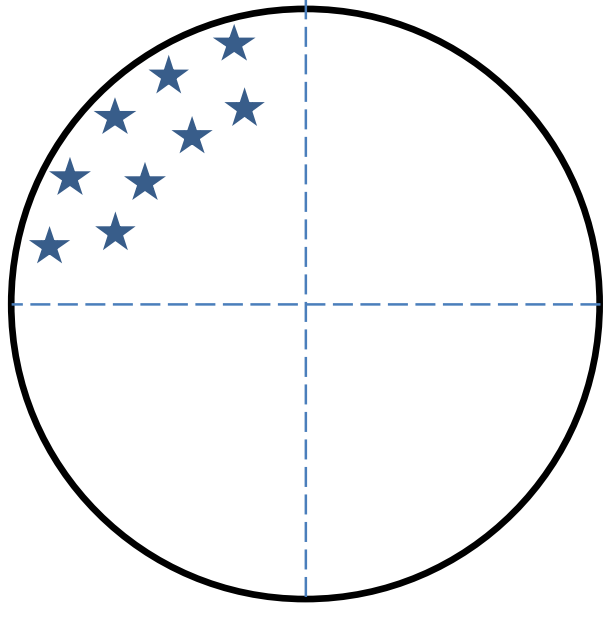

a)

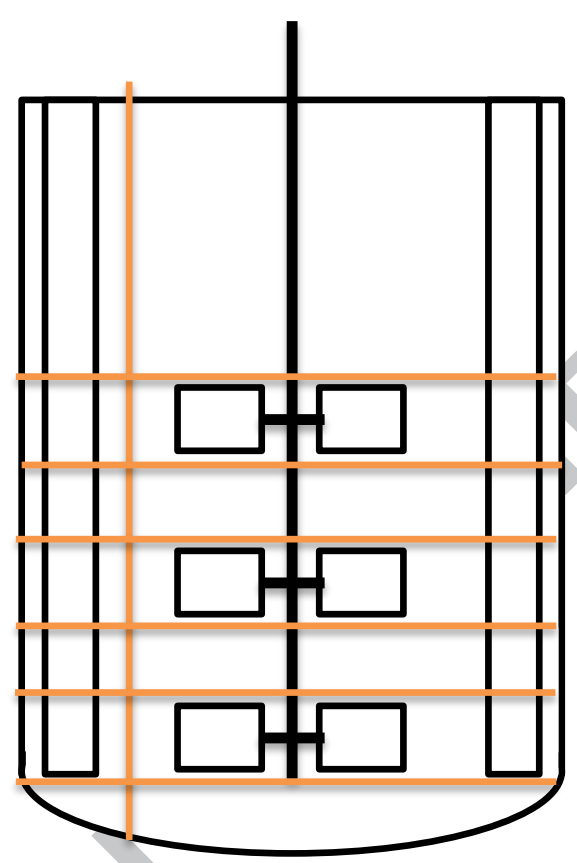

b)

Figure 3: a) Top-down view of the bioreactor. Blue stars indicate where vertical lines have been placed to determine radial flow profiles using evaluation of flows. b) Cross-sectional view of the vertical and horizontal lines, which are used in the method to identify the location of each compartment.

\section{Step 2 - Axial division: Identify axial division at $U_{\text {Rad }}=0$}

The radial flow velocity is evaluated along the vertical lines in the bioreactor. The sign of the radial velocity will indicate whether the bulk flow is directed towards the wall of the bioreactor or towards the impeller shaft. Axial division of the vessel is located at the height of every change in radial flow direction. Figure 4 depicts a common radial velocity profile resulting from this analysis. 


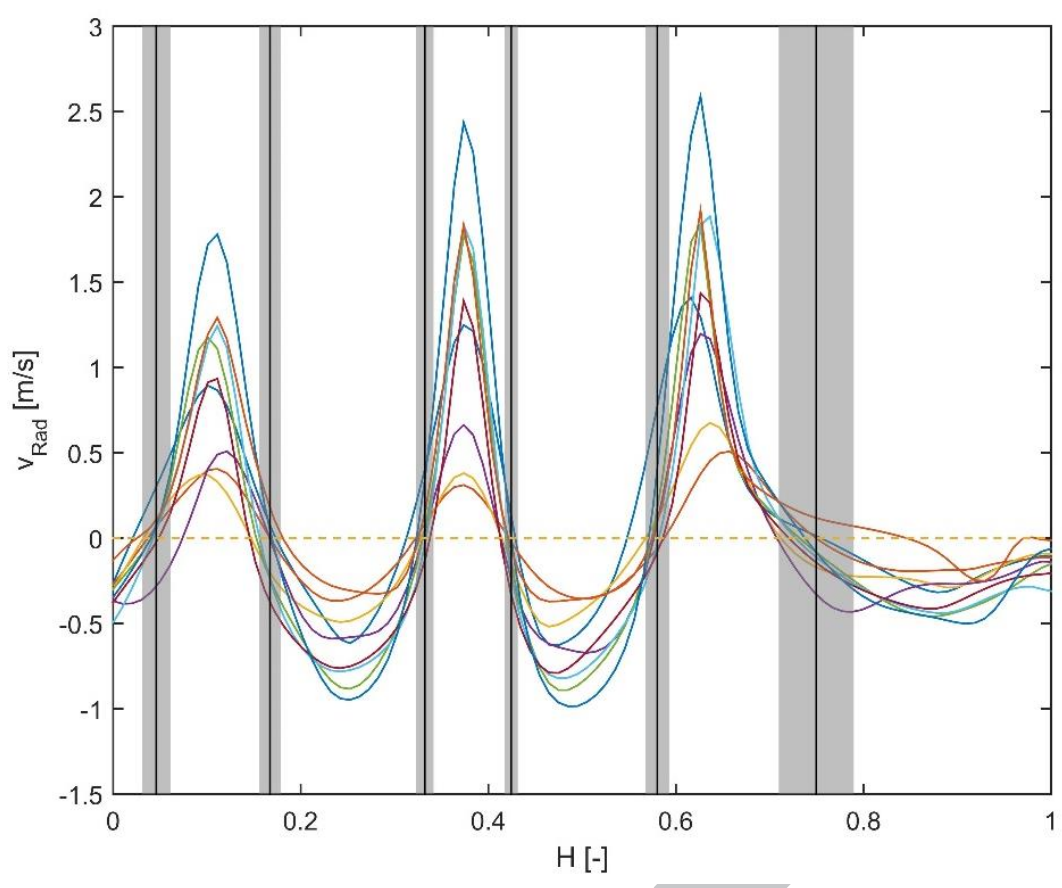

Figure 4: Radial flow profiles at medium agitation speed as a function of dimensionless height evaluated along the vertical lines identified in the first step of the methodology. The black lines indicate the location of axial division and the grey area indicates the standard deviation from the mean division height. The dashed line indicates the turning point from flow towards the wall and towards the shaft.

The collected flow profiles shown in Figure 4 indicate that, despite the varying positions of the vertical lines from Step 1, general trends are observable. The effect of the impeller action is apparent in the peaks of radial velocity for all impeller positions and investigated flow lines. Determination of the axial compartment division is carried out by identifying the reactor height where radial flow alternates from moving towards the reactor wall to moving towards the impeller shaft or vice versa, as indicated by vertical black lines in Figure 4. This results in six axial divisions for the investigated geometry, yielding 7 compartments across the height of the vessel.

\section{Step 3-Radial division: Place horizontal lines at axial divisions}

Based on the axial divisions identified in Step 2 horizontal lines, as shown in Figure 3 b), are placed at each height from the center of the reactor to the wall.

\section{Step 4-Radial division: Determine radial division at $\mathrm{U}_{\text {Axial }}=0$.}

As with Steps 1 to 3, the radial division of compartments is identified by evaluating the axial flow velocity along the horizontal lines resulting from Step 3 in the methodology. The axial velocity profiles along the six horizontal lines are shown in Figure 5. 


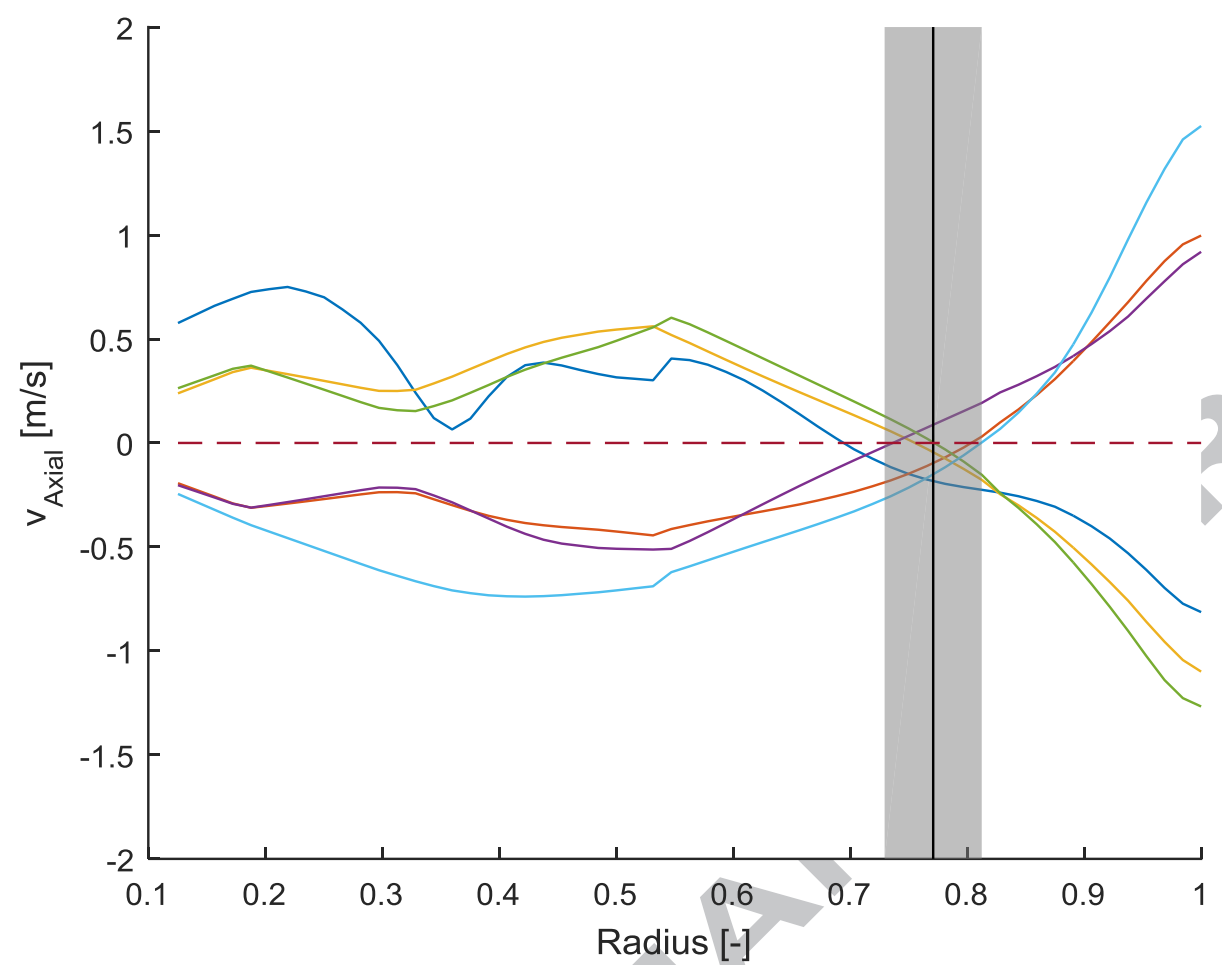

Figure 5: Axial velocity profiles as a function of scaled reactor radius for the six horizontal lines identified in Step 3. The vertical black line indicates the mean radius of flow direction change and the grey area indicates the standard deviation from the mean.

The six velocity profiles shown in Figure 5 can be distributed into two groups, where the first group represents compartments with positive (upward) axial flow close to the impeller and the second group consists of negative (downward) axial flow close to the impeller. The mean intersection radius is used as the definition of the axial division of the vessel.

\section{Circumferential division}

In order to simulate the clockwise mixing of liquid, when seen from above, the vessel is divided into four wedges separated by the planes intersecting each pair of baffles, as illustrated by the blue dashed lines in Figure 3 a).

The full compartmentalization is completed by combining the divisions from Steps 2 and 4 . The resulting compartment structure can be seen in Figure 6 . 


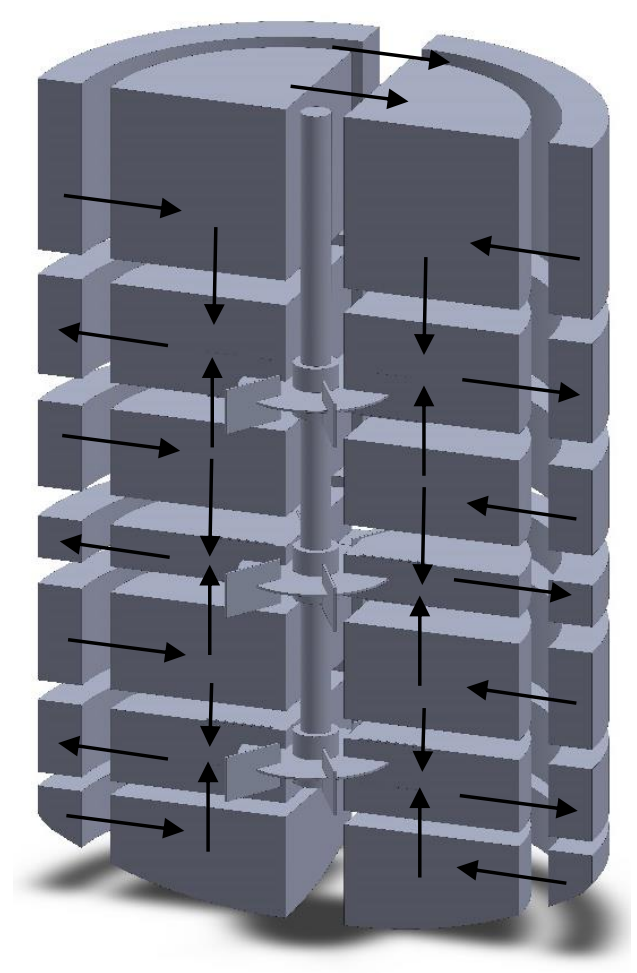

a)

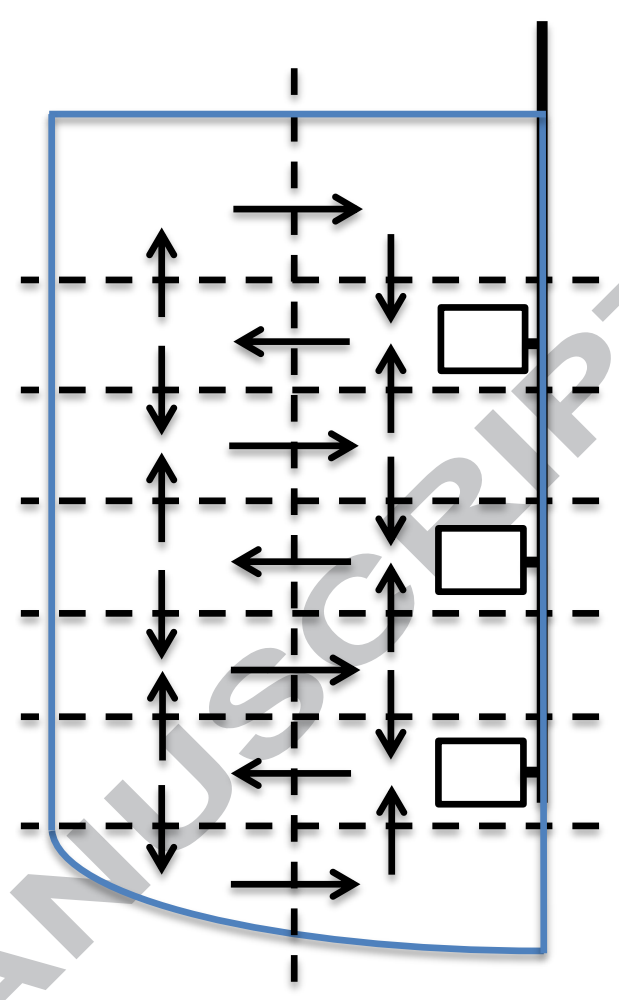

b)

Figure 6: a) Hypothesis-driven compartment model structure. The vessel is divided into 7 axial and 2 radial zones as well as 4 wedges produced by the baffles. The resulting model has 56 compartments. b) $2 \mathrm{D}$ representation of flows in 14 compartments in one wedge. The figure is not to scale.

The structure shown in Figure 6 is similar to previously reported compartment structures for reactors containing multiple RDTs, as it is quite intuitive [16]. However, in contrast to previous attempts, this model structure has been deduced from numerical simulations of the hydrodynamics, and the positions of each compartment have been chosen to ensure that the flows are uniform at the interfaces. A detailed definition of each flow and compartment in the model is described in the Supplementary Information.

\section{Step 5 - Determine flows between identified compartments}

The fifth and final step of the methodology consists of determining the liquid flow between each of the identified compartments resulting from Steps 1 to 4 . The unidirectional flow of every component at each surface is determined as the area integral of the velocity perpendicular to the surface, according to Equation (3.

$$
F=\iint v_{p} d x_{1} d x_{2} \cdot \rho
$$


where $F$ is a unidirectional mass flow, $v_{p}$ is the velocity perpendicular to the given surface, $x_{1}$ and $x_{2}$ are the dimensions suspending the surface and $\rho$ is the liquid density. The integration was carried out in ANSYS CFD-POST, where each interface is defined in the reactor. The data could then be exported in .csv format to be used in MATLAB for further analysis.

In this work, the described methodology has only been applied to a single-phase stirred tank equipped with Rushton turbines, but it is equally applicable to any geometry as well as multiphase systems. It should be noted that, in the investigated case, the circumferential division of the vessel was carried out under the assumption of rotational symmetry. Other geometries, however, might require an investigation of the uniformity of fluid flow in the circumferential direction, along the lines described in Steps 1 and 3.

\section{Results}

In the following section, the experimentally determined tracer profiles are compared with the three model methods discussed in this work. The ability to predict mixing time has previously been used as a measure to benchmark hydrodynamic models, including network-of-zones, CFD and tanks-in-series models [8]. However, if the ultimate aim of a model is to describe process kinetics, such as substrate addition or species mixing, a more rigorous measure should be adopted. In this work, the models have been benchmarked by comparing the predicted and measured $\mathrm{pH}$ tracer profiles from the time of injection to $95 \%$ homogeneous mixing. This is a demanding measure for such models, and has the advantage that it maximizes the utilization of the collected data.

\section{$4+1$ compartment model}

The interchange flows between the four main compartments were estimated using an optimization algorithm based on the tracer response for all agitation speeds and pulse positions. The experimental setup contained five $\mathrm{pH}$ sensors, but the model only contains four bulk compartments, which means that Sensors 1 and 2, at the top of the reactor, are both positioned in the topmost compartment. This is described in Table A2 in the Supplementary Information. The resulting model outputs are summarized in Figure 7. 


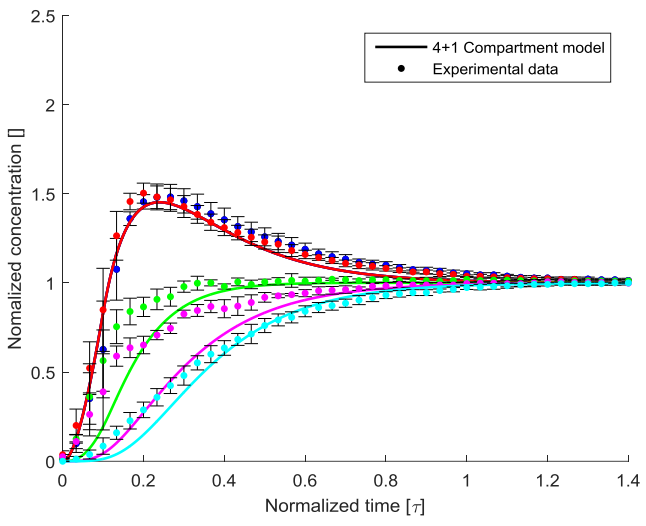

a)

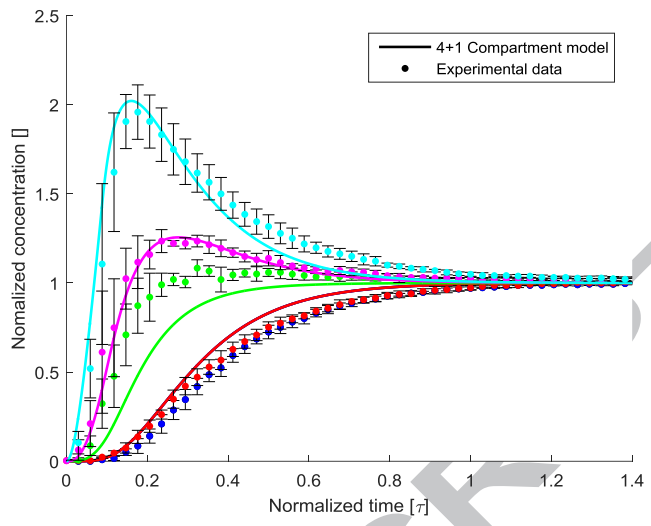

b)

Figure 7: Experimental and simulated tracer response curve for medium agitation using top (a) and bottom dosing (b). Solid lines indicate the model simulations and points depict experimental data.

- Sensor 1, Sensor 2, Sensor 3, Sensor 4 and Sensor 5. The experimental data for the sensors is shown as an average with error bars corresponding to one standard deviation.

Figure 7 a) shows that the $4+1$ compartment model generally has the ability to recreate the tracer profiles of the sensors in the reactor following a three second tracer dose. The fact that the fitted model corresponds well to the experimental data confirms the hypothesis of compartmentalization in reactors with multiple RDTs.

\section{Hypothesis-driven compartment model}

The hypothesis-based compartment method resulted in 56 bulk compartments and a feed compartment, where interchange flows between compartments were calculated from CFD simulations. The predictive ability of the model is evaluated in Figure 8 , where the tracer response from a top and bottom pulse is compared with simulations. 


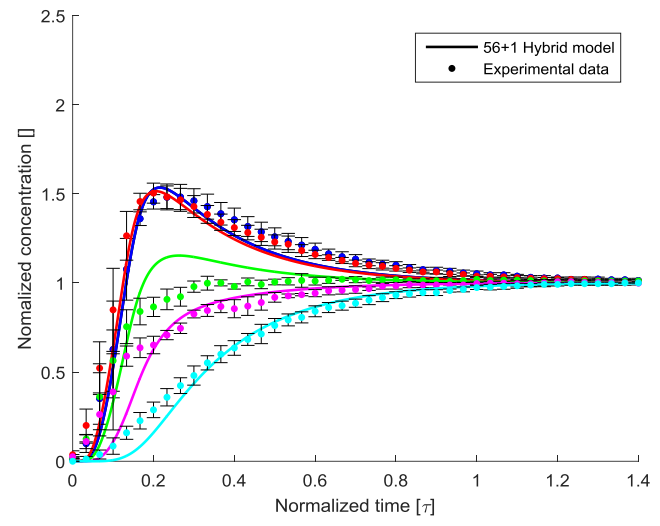

a)

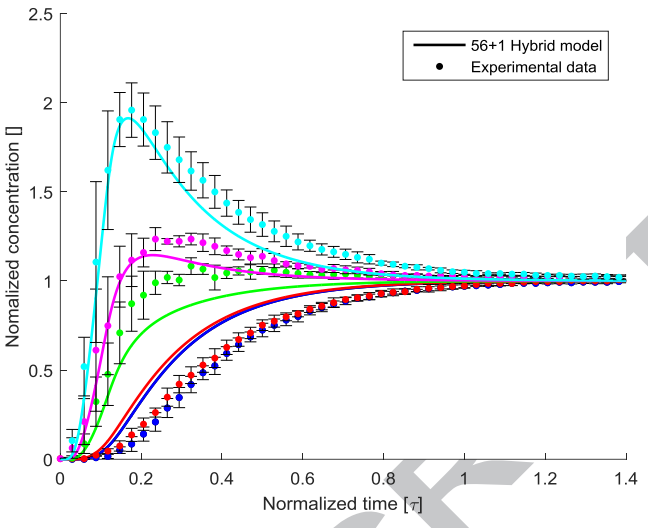

b)

Figure 8: Measured and simulated tracer response from a top a) and bottom b) pulse. The hypothesis-driven compartment model output is shown as a solid line and the data are shown as points. Sensor 1, Sensor 2, Sensor 3, Sensor 4 and Sensor 5 . The experimental data for the sensors is shown as an average with error bars corresponding to one standard deviation.

Following a top pulse, the tracer trajectory of Sensors 1, 2, 4 and 5 are well represented by the model, as shown in Figure 8 a). Compared to the $4+1$ compartment model, the response of Sensors 1 and 2 in the hypothesis-driven approach are two separate outputs. The overshoots of the bottommost sensors (4 and 5) after dosing a pulse from the bottom of the reactor, as depicted in Figure $8 \mathrm{~b}$ ), are also described well by the model. Generally, the developed model describes the transient behavior of tracer addition at both the top and bottom of the reactor very well for all five sensors, and the deviations that can be seen in Figure 8 are mostly small parallel shifts of the response profiles. This indicates that the developed model is able to recreate the major trends of the hydrodynamics occurring in the bioreactors.

\section{CFD model}

In order to benchmark the hypothesis-driven methodology, the ability of the underlying CFD model to represent the dynamic tracer profile was evaluated against the measured values from a top and bottom pulse. The tracer profiles from the CFD simulations, shown in Figure 9, were evaluated at the node closest to the physical position of each sensor in the bioreactor. 


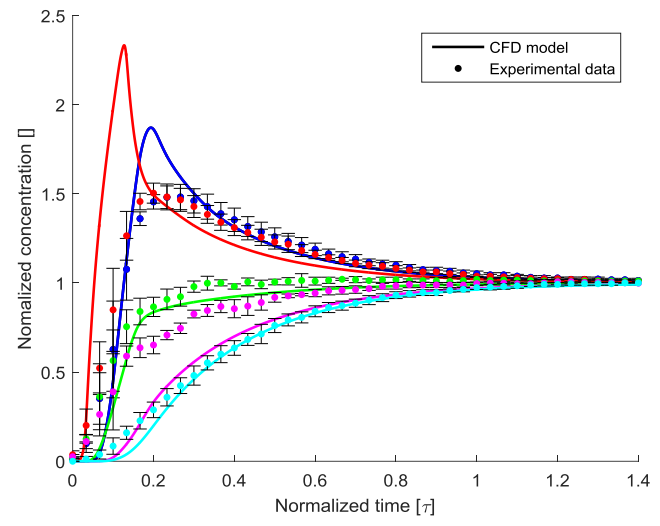

a)

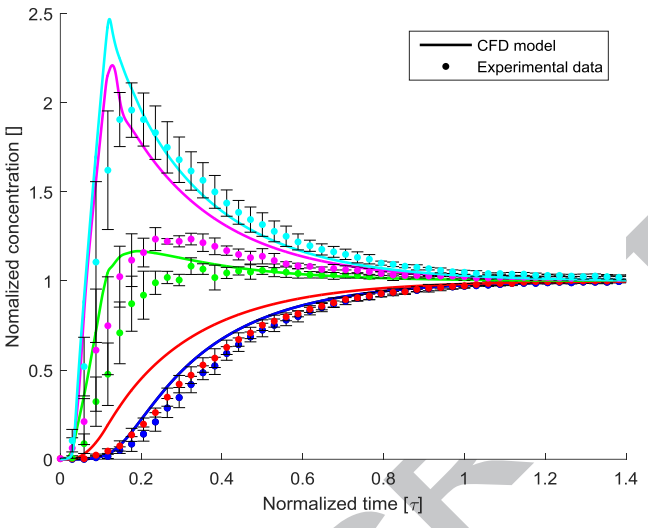

b)

Figure 9: Measured and predicted values of tracer response following a three-second pulse in the top a) and bottom b) of the reactor. The simulations are shown as a solid line and points depict the measurements. Sensor 1, Sensor 2, Sensor 3, Sensor 4 and Sensor 5 . The experimental data for the sensors is shown as an average with error bars corresponding to one standard deviation.

The CFD simulations suggest a larger overshoot of the tracer concentration than observed experimentally for both top and bottom feeding, as seen in Figure 9. The tracer response of Sensor 3 is well described by the CFD simulations in the case of top and bottom dosing. The overshoot observed in the sensor response close to the feeding point is more pronounced than in the other methods investigated above. This is most likely due to the assumption that the tracer is dosed in a perfect pulse. The 4+1 compartment method and the hypothesis-driven approach both include a dosing compartment, which can mitigate the imperfections of the experimental pulse dosing, with a total duration of 3 seconds.

\section{Comparing the models}

In order to compare the performance of the different modeling methods across the different conditions investigated, the root mean square errors (RMSEs) between measurements and predictions were calculated for all conditions as described in Equation (4.

$$
R M S E=\sqrt{\sum \frac{\left(C_{i, j, k}^{e x p}(t)-C_{i, j, k}^{s i m}(t)\right)^{2}}{i * j * k}}
$$

where $C_{i, j, k}(t)$ is the simulated or experimental concentration of tracer measured in compartment $i, j, k$ at time $t$. This measure was scaled by the summated error from the $4+1$ compartment model at 
medium agitation intensity. Furthermore, the RMSE for each condition is scaled by the associated $95 \%$ mixing time to avoid any bias resulting from faster mixing conditions. A summary of this analysis is shown in Table 1.

Table 1: Comparison of compartment model performance based on summed RMSE of each model at different agitation intensities. The summed RMSE values are scaled based on the error of medium agitation of the $4+1$ compartment model.

\begin{tabular}{|c|c|c|c|}
\hline & Low agitation & Medium agitation & High agitation \\
\hline $\mathbf{4 + 1}$ Compartments & 1.37 & 1.00 & 0.78 \\
\hline $\mathbf{5 6 + 1}$ Hypothesis driven & 1.54 & 1.10 & 0.95 \\
\hline CFD & 3.96 & 2.96 & 1.91 \\
\hline
\end{tabular}

Table 1 reveals that the data-driven $4+1$ compartment model predicts the tracer profiles of the $\mathrm{pH}$ sensors more accurately than the hypothesis-driven model and the CFD simulations at all investigated conditions. Furthermore, the models are generally more accurate with increasing agitation intensity, as the reactor approaches ideal mixing. The fact that the simple data-driven $4+1$ compartment model outperforms the other model strategies is unsurprising given the fact that the available data is optimally suited towards model development of this type. Due to the large data pool, the interchange flows between the four bulk compartments can be estimated with minimal correlation, which improves the predictive capabilities. However, most bioreactors described in literature contain only one or two sensors, and thus the collected data would not be sufficient to identify each flow individually and, consequently, the predictive ability of the model would decrease.

The hypothesis-driven compartment model is only $10-12 \%$ worse than the $4+1$ compartment model at the investigated conditions, which is impressive given the fact that all the interchange flows between the 56 compartments were predicted from the original CFD model, i.e. no parameter estimation was performed. In the investigated case study, the performance of the hypothesis-driven methodology is on par with that of the data-driven $4+1$ compartment model, but in cases where less data is available the performance of the data-driven model would be reduced. In contrast, the hypothesis-driven method is less dependent on data and so, it can be assumed that the accuracy showcased in this example can be expected to be similar in other case studies.

It is apparent from Table 1 that the CFD simulation resulted in lower accuracies than the other two models discussed in this work. This is surprising, since the hypothesis-driven model is based on 
results from the CFD simulations. However, the majority of the error associated with the CFD simulations is observed at the beginning of the tracer profiles and originates from the sensor that is placed closest to the feeding location. This may result from the sensitivity of the tracer dispersion and the fact that the compartment models both have a dosing compartment to avoid overestimations.

A comparison of the tracer profiles of each of the models and the associated experimental data is shown in Figure 10.

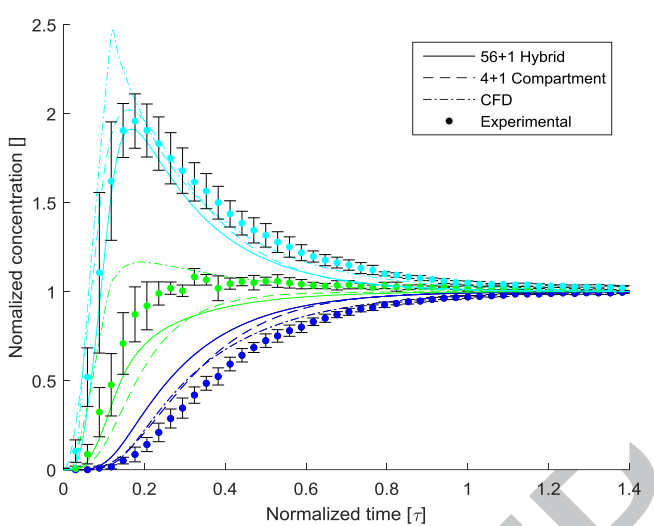

a)

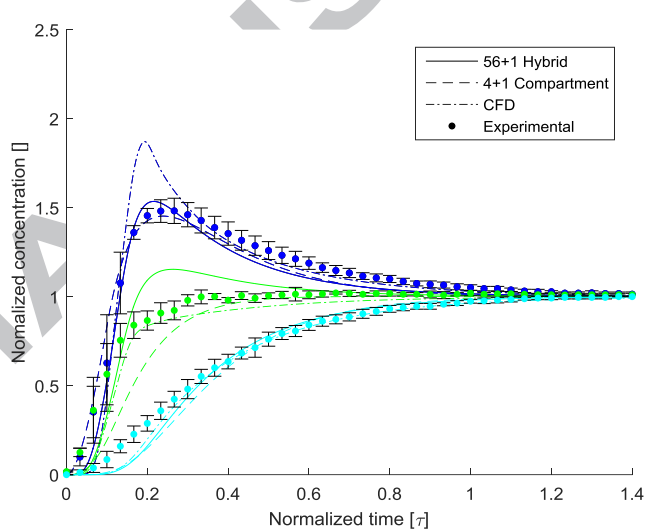

b)

Figure 10: Comparison of experimental data, shown as dots, for - Sensor 1 , Sensor 3 and Sensor 5 and the hypothesis-driven model $(-)$, the $4+1$ compartment model $(--)$ and the CFD model $(-\cdot-)$ following a pulse in the bottom $\mathbf{a}$ ) and top $\mathbf{b})$ of the reactor. The experimental data for the sensors are shown as an average with error bars corresponding to one standard deviation.

The performance of the different models to recreate the tracer response is summarized in Figure 10, and it is apparent that the performance of the models, in terms of tracking the tracer trajectory, is rather similar, with model predictions that are close to the experimental values. As mentioned above, the CFD simulations produce a slightly more pronounced peak in the overshoot of the bottommost sensors, whereas the compartment models provide predictions that are closer to the experimental values. The accuracy of the CFD simulations is similar to previous findings in smaller systems, where CFD was used to predict tracer responses [17]. The model predictions of the trajectory of Sensor 3 in Figure 10 differ between the different model approaches tested, with the CFD simulations predicting a faster increase in concentration than the experimental values, and the compartment models predicting slower approaches towards the homogeneous concentration. For all 
investigated conditions the sensor in the middle of the tank, Sensor 3, has been the most difficult to represent correctly in the model. This could be a result of its position between the middle and topmost impellers, which places it directly between two recirculation loops. Hence, small changes to the position of the loop could greatly affect the sensor response. The accuracy of the model prediction is also affected by the fact that the $\mathrm{pH}$ sensors are not located at the centers of each compartment, and so the measured $\mathrm{pH}$ values may not accurately represent the average $\mathrm{pH}$ in the compartments. However, to confirm whether this is the case, further experiments would have to be done in a setup that allows the positions of the $\mathrm{pH}$ sensors to be adjusted.

The dosing compartment, introduced in the compartment models that have been developed in this work, is a necessary tool required to characterize the feeding of tracer, since experimental dosing is not ideal. The signal measured by the five $\mathrm{pH}$ sensors is highly dependent on the exact feeding characteristic of the dosing pump and tubing, and since the model must recreate this $\mathrm{pH}$ signal in great detail, the dosing compartment is necessary. Depending on the feeding system, the interchange flow between the dosing compartment and the compartment model will change, and so this variable is unique for the setup used, and requires tuning.

Complex phenomena occurring in a stirred bioreactor, whose inclusion in a CFD model simulation are often unfeasible or impractical, due to the resulting excessive simulation time that would be required, can be investigated using the developed compartmentalization method. This has been showcased before, with regards to microbial population heterogeneity, using population balance models $[18,19]$. Furthermore, a simplified compartment model that represents the hydrodynamics of the bioreactor can be used to study multi-dimensional population balance models, which currently cannot be done with typical CFD tools [20]. An especially interesting topic is the developing field of mechanistic fermentation models for monitoring and control of bioprocesses [21]. Correct representation of the hydrodynamics in the fermenter could eliminate the extensive use of process time delays in advanced controllers. In particular, if the manipulated variable were substrate or nutrient feeding the application of an advanced hydrodynamic model would be highly attractive.

It should be noted that, constructing compartment models based on a hypothesis of the hydrodynamics in the process does not only apply to bioreactors. Other processes that rely on complex phenomena and dynamics occurring at a large scale could also benefit from the methodology described in this work.

\section{Experimental recommendations}


The positioning of sensors in a mixing experiment is a balance between systematically positioning the sensors at equidistant intervals, to avoid bias, and placing sensors in critical positions where a bulk-mixing zone is expected, to avoid incorrect assumptions when comparing sensor measurements and compartments in the mathematical models. During the experiments conducted in this work, sensors were positioned systematically and both the sensors and tracer dosing points were placed on the same mounting bracket, for practical reasons. The tracer dosing in the vessel is highly locationdependent, which is why a feeding compartment is used to simulate the addition of tracer to the bulk liquid in the hypothesis-driven and $4+1$ compartment models. In future work, tracer addition should be designed to mimic the substrate addition point in the vessel, to ensure that any assumptions used for tracer feed addition will also apply to substrate addition to achieve better agreement between the pure mixing model and the complete reactor model.

\section{Conclusion}

Pilot scale mixing data was benchmarked against a RANS CFD model and a data-driven four compartment model, representing a highly theoretical and an empirical modelling approach, respectively. Both models were able to describe the mixing data satisfactorily but the models require either large amounts of computational effort or extensive experimental data to be executed. Based on the CFD model, a novel methodology for developing compartment models that describe the hydrodynamics in pilot scale bioreactors has been proposed and evaluated. The methodology utilizes conventional CFD methods to determine the radial and axial placement of each compartment, by evaluating the directional change in axial and radial flow in the bioreactor.

The hypothesis-driven methodology has proven to be within $15 \%$ of the performance of a purely data-driven four-compartment approach for a bioreactor equipped with three Rushton turbines and five $\mathrm{pH}$ sensors. Compared to the data-driven model, the hypothesis-driven model has a larger number of compartments, which enables it to simulate three-dimensional hydrodynamic flow, including simulation of reaction- and mass transfer kinetics, with less severe computational requirements than a typical CFD approach. The model only uses one empirically fitted parameter, which limits the required experimental data significantly in comparison with the data-driven model.

The methodology developed in this work is applicable not only to other bioreactor geometries, but also to entirely different processes such as crystallization, wastewater treatment or combustion, to name a few. Finally, the simplified hydrodynamic model has the potential to serve as a platform for implementation of complex phenomena, which would not be computationally feasible in standard CFD software. 


\section{Acknowledgements}

The authors would like to acknowledge Nanna Petersen Rønnest and Martin Peter Breil who have contributed directly to the project.

This project has received funding from Novo Nordisk A/S, the Technical University of Denmark (DTU) and Innovation Fund Denmark in the frame of the Strategic Research Center BIOPRO2 (BIObased PROduction: TOwards the next generation of optimized and sustainable processes; (Grant number 4105-00020B).

\section{List of references}

[1] A.W. Nienow, Hydrodynamics of Stirred Bioreactors, Appl. Mech. Rev. 51 (1998) 3. doi:10.1115/1.3098990.

[2] N.M. Oosterhuis, N.W. Kossen, Dissolved oxygen concentration profiles in a production-scale bioreactor., Biotechnol. Bioeng. 26 (1984) 546-550. doi:10.1002/bit.260260522.

[3] P. Vrábel, R.G.J.M. Van der Lans, F.N. Van der Schot, K.C. a M. Luyben, B. Xu, S.O. Enfors, CMA: Integration of fluid dynamics and microbial kinetics in modelling of large-scale fermentations, Chem. Eng. J. 84 (2001) 463-474. doi:10.1016/S1385-8947(00)00271-0.

[4] A. Zhang, V.L. Tsang, R. Korke-Kshirsagar, T. Ryll, Effects of pH probe lag on bioreactor mixing time estimation, Process Biochem. 49 (2014) 913-916. doi:10.1016/j.procbio.2014.03.005.

[5] P.A. Vanrolleghem, G. Sin, K. V. Gernaey, Transient response of aerobic and anoxic activated sludge activities to sudden substrate concentration changes, Biotechnol. Bioeng. 86 (2004) 277-290. doi:10.1002/bit.20032.

[6] M. Reuss, Oxygen Transfer and Mixing: Scale-Up Implications, Biotechnol. Set. (2001) 185217. doi:10.1002/9783527620999.ch10b.

[7] R. Mann, R.A. Williams, T. Dyakowski, F.J. Dickin, R.B. Edwards, Development of mixing models using electrical resistance tomography, Chem. Eng. Sci. 52 (1997) 2073-2085. doi:10.1016/S0009-2509(97)00035-3.

[8] C. Bach, J. Yang, H. Larsson, S.M. Stocks, K. V Gernaey, M.O. Albaek, U. Krühne, Evaluation of mixing and mass transfer in a stirred pilot scale bioreactor utilizing CFD, Chem. Eng. Sci. 171 (2017) 19-26. doi:10.1016/j.ces.2017.05.001.

[9] P. Moilanen, M. Laakkonen, J. Aittamaa, Modelling fermenters with CFD, Comput. Aided Chem. Eng. 20 (2005) 709-714. doi:10.1016/S1570-7946(05)80240-8.

[10] F. Bezzo, S. Macchietto, C.C. Pantelides, General hybrid multizonal/CFD approach for bioreactor modeling, AIChE J. 49 (2003) 2133-2148. doi:10.1002/aic.690490821.

[11] A. Delafosse, M.L. Collignon, S. Calvo, F. Delvigne, M. Crine, P. Thonart, D. Toye, CFD-based 
compartment model for description of mixing in bioreactors, Chem. Eng. Sci. 106 (2014) 7685. doi:10.1016/j.ces.2013.11.033.

[12] F. Bezzo, S. Macchietto, A general methodology for hybrid multizonal/CFD models: Part II. Automatic zoning, Comput. Chem. Eng. 28 (2004) 513-525. doi:10.1016/j.compchemeng.2003.08.010.

[13] B.R. Poulsen, J.J.L. Iversen, Mixing determinations in reactor vessels using linear buffers, Chem. Eng. Sci. 52 (1997) 979-984. doi:10.1016/S0009-2509(96)00466-6.

[14] R. Manfredini, V. Cavallera, L. Marini, G. Donati, Mixing and oxygen-transfer in conventional stirred fermenters, Biotechnol. Bioeng. 25 (1983) 3115-3131. doi:10.1002/bit.260251224.

[15] C. Haringa, A.T. Deshmukh, R.F. Mudde, H.J. Noorman, Euler-Lagrange analysis towards representative down-scaling of a $22 \mathrm{~m}^{\wedge} 3$ aerobic S. cerevisiae fermentation, Chem. Eng. Sci. (2017). doi:10.1016/j.ces.2017.01.014.

[16] P. Vrábel, R.G.J.M. Van Der Lans, K.C.A.M. Luyben, L. Boon, A.W. Nienow, Mixing in large-scale vessels stirred with multiple radial or radial and axial up-pumping impellers: Modelling and measurements, Chem. Eng. Sci. 55 (2000) 5881-5896. doi:10.1016/S0009-2509(00)00175-5.

[17] A. Delafosse, M.L. Collignon, S. Calvo, F. Delvigne, M. Crine, P. Thonart, D. Toye, CFD-based compartment model for description of mixing in bioreactors, Chem. Eng. Sci. 106 (2014) 7685. doi:10.1016/j.ces.2013.11.033.

[18] J. Morchain, J.C. Gabelle, A. Cockx, Coupling of biokinetic and population balance models to account for biological heterogeneity in bioreactors, AIChE J. 59 (2013) 369-379. doi:10.1002/aic.13820.

[19] J. Morchain, J.C. Gabelle, A. Cockx, A coupled population balance model and CFD approach for the simulation of mixing issues in lab-scale and industrial bioreactors, AIChE J. 60 (2014) 2740. doi:10.1002/aic.14238.

[20] R.L. Fernandes, M. Carlquist, L. Lundin, A.-L. Heins, A. Dutta, S.J. Sørensen, A.D. Jensen, I. Nopens, A.E. Lantz, K. V. Gernaey, Cell mass and cell cycle dynamics of an asynchronous budding yeast population: Experimental observations, flow cytometry data analysis, and multi-scale modeling, Biotechnol. Bioeng. 110 (2013) 812-826. doi:10.1002/bit.24749.

[21] L. Mears, S.M. Stocks, M.O. Albaek, G. Sin, K. V. Gernaey, Mechanistic Fermentation Models for Process Design, Monitoring, and Control, Trends Biotechnol. 35 (2017) 914-924. doi:10.1016/J.TIBTECH.2017.07.002.

\section{Highlights:}

- Novel hypothesis driven compartment model demonstrated for stirred bioreactor 
- Benchmarked against multiple $\mathrm{pH}$ sensor tracer response in linear $\mathrm{pH}$ buffer

- Comparison with full computational fluid dynamic model 